13,01

\title{
Особенности локализации возбуждений вблизи прослойки между нелинейными фокусирующими средами с нелинейным взаимодействием с границами раздела слоев
}

\section{(C) C.E. Савотченко}

Белгородский государственный технологический университет им. В.Г. Шухова, Белгород, Россия

E-mail: savotchenkose@mail.ru

Поступила в Редакцию 24 июня 2019 г.

В окончательной редакции 24 июня 2019 г.

Принята к публикации 24 октября 2019 г.

В трехслойной структуре, состоящей из двух нелинейных кристаллов с положительной нелинейностью, между которыми зажата линейная пластина конечной толщины, и границы раздела слоев характеризуются нелинейным откликом, существуют локализованные состояния. Получены частоты таких локализованных состояний, которые существуют только при различных значениях интенсивности взаимодействия границ раздела слоев с возбуждениями. Указаны условия существования таких состояний и приведены оценки характерного масштаба локализации поля.

Ключевые слова: граница раздела сред, нелинейная среда, локализованные состояния, нелинейные поверхностные волны, слоистая структура.

DOI: 10.21883/FTT.2020.03.49013.534

\section{1. Введение}

Нелинейные кристаллы широко используются в различных оптоэлектронных устройствах [1-3]. К наиболее распространенным нелинейным кристаллам относятся обладающие эффектом Керра среды, в которых диэлектрическая функция зависит от квадрата амплитуды напряженности электрического поля [4]. Особое значение приобретают композитные многослойные структуры, использующие чередование нелинейных свойств от слоя к слою [5-7]. Возможность управления процессами концентрации потоков энергии полей, происходящих вблизи границ раздела слоев, обусловливает неутихающий интерес к исследованию локализации полей в слоистых структурах $[8,9]$.

При теоритическом изучении таких явлений часто применяется нелинейное уравнение Шредингера (НУШ), содержащее нелинейное, а точнее - кубическое, относительно искомого поля слагаемое [4], описывающее керровскую нелинейность. В последние годы также предлагалось учитывать нелинейные свойства границ раздела слоев [10-23]. Было показано, что нелинейный отклик взаимодействия возбуждений с границами раздела сред привносит новые особенности в процессы формирования локализованных состояний, в том числе в слоистых и периодических структурах [7,24]. Как правило, внутренние свойства границ раздела слоев либо не учитывали, либо учитывались в линейном приближении, причем считались у соседних границ одинаковыми.

Недостаточно изученным остается влияние различия свойств таких границ на особенности локализации состояний. В связи с этим в настоящей работе предлагается теоретическое описание закономерностей локализации возбуждений, обусловленной различным по величине взаимодействием их с границами раздела соседних слоев с нелинейным откликом. Для этого удобно использовать модель, в которой пространственное распределение амплитуды возбуждений поля описывается нелинейным дифференциальным уравнением второго порядка с кубической нелинейностью относительно искомого поля.

К такого вида уравнению сводится стационарное НУШ [15,22,24,25], уравнение для компоненты напряженности электрического/магнитного поля волны с выбранной поляризацией, получаемого из системы уравнений Максвелла [15,21,26], уравнение для обобщенного параметра порядка, получаемого минимизацией функционала свободной энергии в рамках теории ГинзбургаЛандау [5,14,27], уравнение Гросса-Питаевского в теории бозе-эйнштейновской конденсации [27-29]. Поэтому предлагаемая модель может служить универсальной платформой для анализа особенностей формирования локализованных нелинейных состояний различной физической природы.

Моделирование взаимодействия возбуждений поля с границами раздела производится посредством самосогласованного нелинейного потенциала [10-23]. В данной работе интенсивности взаимодействия возбуждений с границами раздела соседних слоев считаются различными.

\section{2. Формулировка модели}

Рассмотрим пластину толщины $2 a$ из линейного кристалла, расположенную между двумя кристаллами, 
характеризующимися фокусирующей (положительной) нелинейностью керровского типа. Систему координат выберем так, чтобы средина линейной прослойки проходила через начало координат. Границы раздела слоев лежат в плоскостях $x= \pm a$ перпендикулярно оси $x$. Нелинейные кристаллы занимают полупространства $|x|>a$, а линейная прослойка расположена в области $|x|<a$.

Для описания интересующих нас свойств и условий их проявления будем использовать одномерную модель на основе стационарного НУШ, которому подчиняется поле $\psi$

$$
\omega \psi=-\frac{1}{2 m} \psi^{\prime \prime}+\Omega(x) \psi-\gamma(x)|\psi|^{2} \psi+U\left(x,|\psi|^{2}\right) \psi,
$$

где $\omega$ - частота стационарных колебаний поля, $\Omega(x)=\Omega_{1}, \quad x<-a, \quad \Omega(x)=\Omega_{L}, \quad|x|<a, \quad \Omega(x)=\Omega_{2}$, $x>a, \Omega_{j}$ - постоянные величины, $j=L, 1,2$, функция керровской нелинейности: $\gamma(x)=\gamma_{1}, x<-a, \gamma(x)=0$, $|x|<a, \gamma(x)=\gamma_{2}, x>a$, где $\gamma_{1,2}$ - постоянные положительные коэффициенты керровской нелинейности, соответствующие фокусирующей нелинейности внешних слоев.

Потенциал, описывающий нелинейные свойства границ раздела, имеет вид

$$
U\left(x,|\psi|^{2}\right)=F_{1}\left(x+a,|\psi|^{2}\right)+F_{2}\left(x-a,|\psi|^{2}\right),
$$

где $F_{j}\left(x,|\psi|^{2}\right)=\left\{U_{j}-W_{j}|\psi|^{2}\right\} \delta(x), j=1,2, U_{j}-$ интенсивности взаимодействия возбуждений с границами раздела в линейном приближении („мощности“ дефектов), $W_{j}$ - параметры нелинейности границ раздела слоев, характеризующие нелинейный отклик их взаимодействия с возбуждениями.

Физическая интерпретация модели с НУШ (1) приводилась ранее неоднократно в предыдущих работах. К примеру, в [22] возмущение намагниченности описано на основе НУШ (1) с нелинейным потенциалом (2), моделирующим границы раздела ферромагнитных слоев в трехслойной структуре, в которой слои одноосных ферромагнетиков и тонкопленочные границы их раздела характеризуются магнитной анизотропией. В $[12,15,21]$ НУШ (1) использовано для описания локализации электрического поля в световом потоке вдоль границ раздела с нелинейными свойствами, разделяющих нелинейные оптические среды с одинаковыми или противоположными знаками нелинейности, либо линейные и нелинейные среды. В $[14,17]$ НУШ (1) использовано для описания электронных фазовых переходов в неоднородные состояния, приводящих к возникновению локализованных состояний типа волн спиновой плотности рамках в модели зонного антиферромагнетика с конгруэнтными сечениями поверхности Ферми, где $\psi$ представляет собой обобщенный параметр порядка, описывающий распределение линейно поляризованных волн спиновой плотности.

В отличие от [22], где все три слоя считались нелинейными, в данной работе рассматривается случай, когда внутренний слой представляет собой линейную пластину между двумя нелинейными кристаллами. Подобная структура с линейной прослойкой в нелинейном кристалле рассматривалась в [30], однако она считалась симметричной по всем свойствам слоев и границ, а в данной роботе свойства границ считаются различными, что приводит к появлению локализованных состояний с новыми свойствами, формирование которых характерно только для таких трехслойных структур, границы в которых взаимодействуют с возбуждениями с различными интенсивностями. В [31] были показано, что имеются особые поверхностные волны, существующие только в случае различных значений параметров границ раздела слоев, в том числе и их нелинейных откликов, в волноводе, который представлял собой нелинейную самофокусирующую оптическую прослойку в линейной среде.

Решение НУШ (1) с потенциалом (2) эквивалентно решению НУШ без потенциала с граничными условиями

$$
\begin{gathered}
\psi( \pm a-0)=\psi( \pm a+0), \\
\psi^{\prime}( \pm a+0)-\psi^{\prime}( \pm a-0)=2 m\left\{U_{j}-W_{j}|\psi( \pm a)|^{2}\right\} \psi( \pm a) .
\end{gathered}
$$

Здесь и далее значение индекса $j=1$ соответствует величинам, относящимся к области $x<-a$, а $j=2-$ к области $x>a$. В (4) и далее для $j=1$ следует выбирать нижний знак, а для $j=2-$ верхний.

\section{3. Локализованные состояния первого типа}

Решение НУШ (1) представим в виде: $\psi(x)=\psi_{1}$, $x<-a, \psi(x)=\psi_{L},|x|<a, \psi(x)=\psi_{2}, x>a$.

Если частота волны лежит в диапазоне $\omega<\min \left\{\Omega_{j}\right\}$, то в нелинейных внешних средах с фокусирующей нелинейностью локализованные состояния первого типа описываются решениями НУШ (2) вида

$$
\psi_{j}(x)=q_{1} / \sqrt{m \gamma_{j}} \operatorname{ch} q_{j}\left(x \mp a \pm x_{j}\right),
$$

а во внутренней прослойке

$$
\psi_{L}(x)=A \exp \left(q_{L} x\right)+B \exp \left(-q_{L} x\right),
$$

где $q_{j}=\sqrt{2 m\left(\Omega_{j}-\omega\right)}$.

После подстановки (5) и (6) в граничные условия (3) и (4) получаются амплитуды $A$ и $B$ и положения „центров“" $x_{j}$

$$
\begin{gathered}
A=\frac{1}{2 \sqrt{m} \operatorname{sh}\left(2 q_{L} a\right)}\left(\frac{q_{2} e^{q_{L} a}}{\sqrt{\gamma_{2}} \operatorname{ch}\left(q_{2} x_{2}\right)}-\frac{q_{1} e^{-q_{L} a}}{\sqrt{\gamma_{1}} \operatorname{ch}\left(q_{1} x_{1}\right)}\right), \\
B=\frac{1}{2 \sqrt{m} \operatorname{sh}\left(2 q_{L} a\right)}\left(\frac{q_{1} e^{q_{L} a}}{\sqrt{\gamma_{1}} \operatorname{ch}\left(q_{1} x_{1}\right)}-\frac{q_{2} e^{-q_{L} a}}{\sqrt{\gamma_{2}} \operatorname{ch}\left(q_{2} x_{2}\right)}\right), \\
\frac{\eta q_{1} e^{-2 q_{L} a} \operatorname{ch}\left(q_{2} x_{2}\right)}{q_{2} \operatorname{ch}\left(q_{1} x_{1}\right)}=\frac{a_{L}+\Delta_{2}}{q_{L}-\Delta_{1}}=\frac{q_{L}-\Delta_{2}}{q_{L}+\Delta_{1}} e^{-2 q_{L} a},
\end{gathered}
$$

где $\eta=\sqrt{\gamma_{2} / \gamma_{1}}, \Delta_{j}=q_{j} \operatorname{th}\left(q_{j} x_{j}\right)+u_{j}-w_{j} q_{j}^{2} / \operatorname{ch}^{2}\left(q_{j} x_{j}\right)$, $u_{j}=2 m U_{j}, w_{j}=2 W_{j} / \gamma_{j}$. 
Из (9) определяются положения „центров“ $x_{j}$ как функции параметров слоистой структуры, затем они подставляются в (7) и (8), в результате чего через характеристики слоистой структуры будут определены все параметры локализованного состояния (5), (6). Такое локализованное состояние существует при допустимых значениях частоты $\omega<\min \left\{\Omega_{j}\right\}$.

Если максимумы амплитуд локального состояния приходятся на границы слоев, когда $x_{j}=0$, то такое локализованное состояние существует только при определенной зависимости частоты от параметров слоистой структуры. Если дополнительно положить $\Omega_{1}=\Omega_{2}=\Omega$, то из (9) в этом случае удается получить точное решение дисперсионного уравнения, которое определяет частоту локализации возбуждений в виде

$$
\omega=\Omega-\Omega_{A}\left\{1 \pm\left(1+\Omega_{B} / \Omega_{A}\right)^{1 / 2}\right\}^{2},
$$

где

$$
\begin{aligned}
& \Omega_{A}=\left[2\left(u_{2} w_{2}-u_{1} w_{1} \eta^{2}\right)+1-\eta^{2}\right] / 4 m\left(w_{2}^{2}-w_{1}^{2}\right), \\
& \Omega_{B}=\left(u_{1}^{2} \eta^{2}-u_{2}^{2}\right) / m\left[2\left(u_{2} w_{2}-u_{1} w_{1} \eta^{2}\right)+1-\eta^{2}\right] .
\end{aligned}
$$

Локализованное состояние с частотой (10) существует при выполнении условия

$$
\eta^{2}<\frac{2 u_{2}\left(w_{2}+u_{2} \sqrt{\left|w_{1}^{2}-w_{2}^{2}\right|}\right)+1}{2 u_{1}\left(w_{1}+u_{1} \sqrt{\left|w_{1}^{2}-w_{2}^{2}\right|}\right)+1},
$$

которое определяет ограничение для коэффициентов керровской нелинейности в зависимости от характеристик границ раздела слоев.

В приближении слабого (но конечного) нелинейного отклика, когда $w_{j} q^{2} \ll u_{j}$, из дисперсионного уравнения получается оценка частоты локализации с максимумами на границах

$$
\omega=\Omega-\left(u_{2}^{2}-u_{1}^{2} \eta^{2}\right) / 2 m\left[2\left(u_{2} w_{2}-u_{1} w_{1} \eta^{2}\right)+1-\eta^{2}\right] .
$$

Для локализации возбуждения в состояние с частотой (11) должны выполняться условия: $\eta^{2}>u_{2}^{2} / u_{1}^{2}$ и $\eta^{2}>\left(1+2 u_{2} w_{2}\right) /\left(1+2 u_{1} w_{1}\right)$ или оба противоположных неравенства.

\section{4. Локализованные состояния второго типа}

В рассматриваемой слоистой структуре существует локализованное состояние второго типа, когда частота волны лежит в диапазоне $\Omega_{L}<\omega<\min \left\{\Omega_{1,2}\right\}$. В нелинейных внешних средах с фокусирующей нелинейностью распределения поля в локализованном состоянии второго типа описываются выражением (5), а во внутренней прослойке -

$$
\psi_{L}(x)=A \sin (k x)+B \cos (k x),
$$

где $k=\sqrt{2 m\left(\omega-\Omega_{L}\right)}$.
После подстановки (5) и (12) в граничные условия (3) и (4) получаются амплитуды $A$ и $B$ и положения „центров“" $x_{j}$

$$
\begin{gathered}
A=\frac{1}{2 \sqrt{m} \cos (k a)}\left(\frac{q_{1}}{\sqrt{\gamma_{1}} \operatorname{ch}\left(q_{1} x_{1}\right)}+\frac{q_{2}}{\sqrt{\gamma_{2}} \operatorname{ch}\left(q_{2} x_{2}\right)}\right), \\
B=\frac{1}{2 \sqrt{m} \sin (k a)}\left(\frac{q_{2}}{\sqrt{\gamma_{2}} \operatorname{ch}\left(q_{2} x_{2}\right)}-\frac{q_{1}}{\sqrt{\gamma_{1}} \operatorname{ch}\left(q_{1} x_{1}\right)}\right), \\
\frac{\eta q_{1} \operatorname{ch}\left(q_{2} x_{2}\right)}{q_{2} \operatorname{ch}\left(q_{1} x_{1}\right)}=\frac{k \operatorname{ctg}(k a)-\Delta_{2}}{k \operatorname{ctg}(k a)+\Delta_{1}}=\frac{\Delta_{2}+k \operatorname{tg}(k a)}{\Delta_{1}-k \operatorname{tg}(k a)} .
\end{gathered}
$$

Так же как и для состояний первого типа, из (15) определяются положения ,центров“ $x_{j}$, затем они подставляются в (13) и (14), в результате чего через характеристики слоистой структуры будут определены все параметры локализованного состояния второго типа $(5),(12)$, которое существует при допустимых значениях частоты $\Omega_{L}<\omega<\min \left\{\Omega_{1,2}\right\}$.

Частота локализации возбуждений с максимумами амплитуд на границах слоев, когда $x_{j}=0$ и $\Omega_{1}=\Omega_{2}=\Omega$, для состояний второго типа также представима в виде (10), но с другими параметрами

$$
\begin{gathered}
\Omega_{A}=\left[2\left(u_{1} w_{1} \eta^{2}-u_{2} w_{2}\right)-1+\eta^{2}\right] / 4 m\left(w_{1}^{2} \eta^{2}-w_{2}^{2}\right), \\
\Omega_{B}=\left[2 m\left(\Omega-\Omega_{0}\right)\left(1-\eta^{2}\right)-\eta^{2}\left(u_{1}^{2}-u_{2}^{2}\right)\right] / \\
m\left[2\left(u_{1} w_{1} \eta^{2}-u_{2} w_{2}\right)-1+\eta^{2}\right] .
\end{gathered}
$$

Условие существования такого локализованного состояния можно сформулировать в виде

$$
\begin{aligned}
\Omega> & \Omega_{0}+\frac{1}{2 m\left(1-\eta^{2}\right)} \\
& \times\left\{\eta^{2}\left(u_{1}^{2}-u_{2}^{2}\right)-\frac{\left[2\left(u_{1} w_{1} \eta^{2}-u_{2} w_{2}\right)-1+\eta^{2}\right]}{4\left(w_{1}^{2} \eta^{2}-w_{2}^{2}\right)}\right\} .
\end{aligned}
$$

Для слабого нелинейного отклика, когда $w_{j} q^{2} \ll u_{j}$, можно получить оценку частоты локализации с максимумами на границах

$$
\begin{array}{r}
\omega=\Omega-\left\{\eta^{2}\left(u_{1}^{2}-u_{2}^{2}\right) / 2 m-\left(\Omega-\Omega_{0}\right)\left(1-\eta^{2}\right)\right\} / \\
{\left[2\left(u_{1} w_{1} \eta^{2}-u_{2} w_{2}\right)-1+\eta^{2}\right] .}
\end{array}
$$

\section{5. Обсуждение результатов}

Следует отметить, что локализованные состояния обоих типов с частотами (10) существуют также и при одинаковых нелинейностях слоев (когда $\eta=1$ ), но при различных характеристиках их границ раздела. При этом обязательным условием является наличие различных нелинейных откликов взаимодействия возбуждений с границами, так как при одинаковых или нулевых значениях $W_{j}$ такие состояния не реализуются.

При одинаковых нелинейностях слоев для слабого нелинейного отклика (когда $\eta=1$ и $\left.w_{j} q^{2} \ll u_{j}\right)$ из (11) 
и (16) получается одинаковая зависимость частоты от параметров структуры

$$
\omega=\Omega-\left(u_{2}^{2}-u_{1}^{2}\right) / 4 m\left(u_{2} w_{2}-u_{1} w_{1}\right) .
$$

Характерный масштаб локализации возбуждений с частотой (17) вблизи прослойки, то есть глубина проникновения поля во внешние слои структуры, оценивается выражением

$$
l=1 / q=\left\{2\left(u_{2} w_{2}-u_{1} w_{1}\right) /\left(u_{2}^{2}-u_{1}^{2}\right)\right\}^{1 / 2} .
$$

Из (18) следует, что увеличения глубины проникновения поля можно добиться подбором близких друг другу величин „мощностей дефектов“ двух границ трехслойной структуры. Получается, что управление локализацией поля вдоль прослойки слоистой структуры на определенной частоте может осуществляться регулировкой параметров границ раздела слоев.

В частности, если интенсивность взаимодействия одной из границ с возбуждениями намного больше, чем с другой (а нелинейные отклики одного порядка), для определенности, к примеру, $u_{1} \gg u_{2}$, то из (18) получается, что глубина проникновения поля в основном порядке оценивается по формуле: $l=\left(2 W_{1} / m \gamma_{1} U_{1}\right)^{1 / 2}$. Следовательно, увеличение интенсивности нелинейного отклика границы приводит к уширению характерного масштаба локализации возбуждений. К такому же эффекту приводит уменьшение „мощности дефекта“ и снижение коэффициента керровской нелинейности.

Если теперь считать, что нелинейный отклик одной из границ намного больше (а „мощности дефекта“ одного порядка), чем с другой, для определенности, к примеру, $w_{1} \gg w_{2}$, то в основном порядке из (18) можно получить оценку глубины проникновения поля: $l=\left\{2 u_{1} w_{1} /\left(u_{1}^{2}-u_{2}^{2}\right)\right\}^{1 / 2}$, причем обязательно $u_{1} \neq u_{2}$.

Для существования локализованных состояний первого типа с частотой (17) достаточно либо выполнения следующих неравенств $\left|u_{1} / u_{2}\right|>1$ и $u_{1} w_{1}>u_{2} w_{2}$ либо им противоположных (так как должно быть $l>0$ ). Для существования локализованных состояний второго типа эти условия дополняются еще одним требованием: $\Omega-\Omega_{0}>1 / 2 m l^{2}$. В обоих случаях видно, что такие состояния не реализуются даже при одинаковых „мощностях дефектов“" (когда $U_{1}=U_{2}$ ) или при отсутствии нелинейного отклика (когда $\left.W_{j}=0\right)$.

\section{6. Заключение}

Таким образом, в данной работе на основе модели трехслойной структуры с границами разделов, характеризующимися взаимодействием с возбуждениями, в том числе и нелинейного характера, показана возможность существования таких локализованных состояний, которые реализуются только при различных интенсивностях взаимодействия границ раздела слоев с возбуждениями, и при этом нелинейность взаимодействия играет решающую роль.
Получены оценки глубины проникновения поля в кристалл при удалении от прослойки. Показано, что увеличение характерного масштаба локализации возбуждений происходит вследствие роста интенсивности нелинейного отклика границ. К такому же эффекту приводит уменьшение „мощности дефекта“ и снижение коэффициента керровской нелинейности. Регулировка значений параметров границ раздела слоев позволяет контролировать масштаб локализации поля вдоль прослойки слоистой структуры на фиксированной частоте.

Полученные в работе результаты могут иметь значение при проектировании элементов электронных устройств, основанных на использовании свойств поверхностных волн и контролированной локализации потоков энергии. Закономерности формирования несимметричного распределения поля вдоль границ раздела слоистых структур важны для расчетов значений характеристик интерфейсов, управляющих режимами переключения пропускания и запирания потоков полей $[32,33]$.

\section{Конфликт интересов}

Авторы заявляют, что у них нет конфликта интересов.

\section{Список литературы}

[1] T. Strudley, R. Bruck, B. Mills, O. Muskens. Light: Sci. Appl. 3, e207 (2014).

[2] B.A. Naim. Chinese J. Phys. 55, 2384 (2017).

[3] G. Bettella, R. Zamboni, G. Pozza, A. Zaltron, C. Montevecchi, M. Pierno, G. Mistura, C. Sada, L. Gauthier-Manuel, M. Chauvet. Sensors Actuat. B 282, 391 (2019).

[4] Ю.С. Кившарь, Г.П. Агравал. Оптические солитоны. От волоконных световодов до фотонных кристаллов. Физматлит, М. (2005) 648 с.

[5] В.Н. Меньшов, В.В. Тугушев. ФТТ 44, 1650 (2002).

[6] И.В. Герасимчук, А.С. Ковалев. ФТТ 45, 1088 (2003).

[7] Y.V. Kartashov, B.A. Malomed, L. Torner. Rev. Mod. Phys. 83, 247 (2011).

[8] B.G. Luther-Davies, I. Stegeman. Materials for spatial solitons. In: Spatial Optical Solitons/ eds S. Trillo, W.E. Torruellas. Springer-Verlag, N.Y. (2001). P. 19.

[9] R. Carretero-González, J. Cuevas-Maraver, D. Frantzeskakis, N. Karachalios, P. Kevrekidis, F. Palmero-Acebedo. Localized Excitations in Nonlinear Complex Systems. Springer Science \& Business Media. (2013). 432 p.

[10] E. Lidorikis, K. Busch, L. Qiming, C.T. Chan, C.M. Soukoulis. Phys. Rev. B 56, 15090 (1997).

[11] И.В. Герасимчук. ЖЭТФ 121, 596 (2015).

[12] S.E. Savotchenko. Mod. Phys. Lett. B 32, 1850120 (2018).

[13] S.E. Savotchenko. Mod. Phys. Lett. B 32, 1850371 (2018).

[14] С.Е. Савотченко. Письма в ЖЭТФ 107, 481 (2018).

[15] С.Е. Савотченко. Письма в ЖЭТФ 108, 175 (2018).

[16] С.Е. Савотченко. ЖЭТФ 154, 517 (2018).

[17] S.E. Savotchenko. Solid State Commun. 283, 1 (2018).

[18] S.E. Savotchenko. Surf. Interfaces 13, 157 (2018).

[19] С.Е. Савотченко. Изв. вузов. Физика 62, 3 (2019).

[20] С.Е. Савотченко. ФТТ 61, 571 (2019). 
[21] С.Е. Савотченко. ФТТ 61, 626 (2019).

[22] С.Е. Савотченко. ФТТ 61, 698 (2019).

[23] S.E. Savotchenko. Surf. Interfaces 15, 191 (2019).

[24] A.A. Sukhorukov, Yu.S. Kivshar. J. Opt. Soc. Am. B 19, 772 (2002).

[25] I.V. Gerasimchuk, V.S. Gerasimchuk. J. Appl. Phys. 124, 085301 (2018).

[26] Д. Михалаке, Р.Г. Назмитдинов, В.К. Федянин. Физика элементарных частиц и атомного ядра 20, 198 (1989).

[27] В.Н. Меньшов, В.В. Тугушев. ЖЭТФ 120, 899 (2001).

[28] А.В. Чаплик. Письма в ЖЭТФ 105, 565 (2017).

[29] Н.В. Высотина, Н.Н. Розанов, А.Н. Шацев. Оптика и спектроскопия 124, 82 (2018).

[30] S.E. Savotchenko. Mod. Phys. Lett. B 33, 1950127 (2019).

[31] С.Е. Савотченко. Оптика и спектроскопия 127, 159 (2019).

[32] M.D. Tocci, M.J. Bloemer, M. Scalora, J.P. Dowling, C.M. Bowden. Appl. Phys. Lett. 66, 2324 (1995).

[33] S. Lan, H. Ishikawa. J. Appl. Phys. 91, 2573 (2002).

Редактор Ю.Э. Китаев 